\title{
KANT Y EL PROBLEMA DE LA GEOMETRÍA
}

\author{
José Manuel Osorio
}

Pontificia Universidad Católica del Perú

Resumen: Para Kant la geometría es una disciplina matemática que contiene proposiciones y juicios sintéticos a priori. Sin embargo, esta afirmación no se encuentra libre de problemas. La intención del artículo será mostrar I) cómo entiende Kant la apodicticidad, universalidad y sinteticidad de la geometría en la Crítica de la razón pura; y 2) qué relevancia tiene hoy en día estudiar la teoría kantiana de la geometría luego de la superación de la teoría euclidiana del espacio. Con respecto a (I): Kant entiende a la geometría como la ciencia que objetiva la intuición pura del espacio. Todo concepto geométrico se construye en la intuición del espacio mediante un proceso sintético que exhibe la figura geométrica. Además, la intuición pura del espacio es la forma del sentido externo. Por tanto, los objetos geométricos y los fenómenos externos comparten un territorio común: el espacio como intuición pura. Este aspecto común garantiza la universidad de la geometría. Con respecto a (2): la importancia de estudiar la teoría kantiana de la geometría no solo radica en que esta disciplina determina $a$ priori su objeto y por tanto sirve de ejemplo a la filosofía, sino que la comprensión del objeto de la geometría, el espacio como intuición pura, nos obliga a pasar revista a lo qué entiende Kant por sensibilidad y su relación con el espacio. El estudio de la sensibilidad obliga a Kant a repensar qué se entiende por espacio y, con ello, qué se entiende por geometría. El análisis de la teoría kantiana de la geometría, entonces, equivale al estudio de la teoría kantiana de la sensibilidad.

Palabras clave: Geometría, matemática, espacio, sensibilidad, a priori.

Abstract: Geometry is an a priori science. However, its apriority is saddled with problems. The aim of this paper will be to show I) how Kant understands that the contents of geometry are synthetic a priori judgments in the Critique of Pure Reason, and 2) if it's still relevant to study Kant's theory of geometry after the challenges posed by nonEuclidian theories of space. With respect to point I: Kant understands geometry as the 
discipline that objectifies the pure intuition of space. Every geometric concept is built upon the pure intuition of space through a synthetic ostensive process. Furthermore, the pure intuition of space is the form of external experiences. Thus, geometry and external phenomena share a common ground - pure space. This common ground is what provides an answer to the question of the possibility of mathematics as a universal and a priori science. With respect to point 2: the relevance of studying Kant's theory of geometry lies not only in the fact that geometry can serve as an example to philosophy based on the fact that it establishes its propositions a priori, but also because the object-study of geometry - the pure intuition of space-forces the reader to review Kant's thoughts about sensibility and its relation to space. The analysis of Kant's theory of geometry then amounts to studying Kant's theory of sensibility.

Key Words: Geometry, Mathematics, Space, Sensibility, A priori.

\section{§ 1. Introducción}

El título de este trabajo no ha sido elegido sin previa consideración. En efecto, la concepción kantiana de la geometría no se encuentra libre de problemas ni en el plano intratextual, es decir, los diversos argumentos al interior del corpus kantiano sobre geometría parecen, según algunos especialistas, no seguirse unos de otros, ni tampoco en un plano puramente científico, pues la aparición de nuevas teorías acerca del espacio ha puesto en cuestión seriamente el alcance explicativo de la teoría kantiana ${ }^{130}$.Si la teoría kantiana de la geometría parece haber sido "superada" completamente hace un considerable número de años, vale la pena preguntarse qué relevancia tiene hoy en día su estudio; ¿es, como se podría pensar en primera instancia, debido a una mera curiosidad de anticuario? ¿O su estudio nos provee información valiosa no solo acerca del estatus de la investigación sobre geometría en la ciencia y la filosofía del siglo XVIII, sino además acerca del significado del concepto

I30 En concreto, esta posición descansa sobre una concepción dicotómica acerca del estatuto epistemológico de las distintas teorías sobre la naturaleza del espacio: o bien el espacio obedece a las leyes euclidianas o bien a las no-euclidianas. Si una es verdadera, necesariamente la otra teoría no lo será. Por lo tanto, al ser el espacio no-euclidiano, la teoría kantiana de la geometría, en esencia euclidiana, no es más verdadera. Pero esta respuesta no parece ser tan evidente ni intuitiva, ya sea desde la geometría misma o desde la filosofía de la ciencia. Sobre el problema, cf. Gray J., Worlds Out of Nothing. A Course in the History of Geometry in the 19th Century, Londres: Springer, pp. 325-330; también cf. Trudeau, R., The Non-Euclidean Revolution, Berlín, Boston: Birkhauser, 1987, pp. 248-259. 
"ciencia" en Kant? Al hablar de geometría en Kant, entonces, lo primero que debe hacerse es diferenciar no solo los tipos de pregunta que realizamos al estudiarla, sino además desde dónde realizamos estas preguntas. De un lado, se interroga historiográficamente el texto por el lugar y valor de estos argumentos en la obra de Kant y en su horizonte histórico; de otro lado, se pregunta científico-positivistamente si estos argumentos tienen alguna validez científica hoy en día. Este trabajo se ocupará del problema de la geometría en Kant desde la primera perspectiva. Lo que nos interesa es comprender qué valor e importancia tiene la argumentación kantiana sobre la geometría al interior de su obra crítica.

Para Kant, la geometría es la ciencia que objetiva el espacio ${ }^{131}$. ¿En qué tipo de espacio piensa Kant? Él sostiene que el espacio es una intuición pura que lo representa de manera única y singular. Sin embargo, icómo es posible que la geometría se ocupe del espacio único y singular y, al mismo tiempo, de diferentes espacios geométricos (si consideramos a los objetos geométricos como partes del espacio)? Más importante, icómo se convierte a una intuición pura en objeto, a su vez, de intuición, de modo que la geometría pueda ser ciencia y ocuparse del espacio?

En el prólogo a la segunda edición ${ }^{132}$ de la Crítica de la razón pura, Kant argumenta a favor de la investigación filosófica sobre la geometría y la posibilidad de sus juicios sintéticos a priori. Sostiene que la matemática y la física son dos conocimientos teóricos que deben $-y$, de hecho, pueden- determinar a priori sus objetos; la primera, la matemática, y como parte de ella la geometría, de manera completamente a priori. Kant presenta como caso paradigmático de la determinación a priori del objeto en la ciencia un ejemplo tomado de la geometría. Según Kant, la geometría anduvo por camino seguro desde que un geómetra descubrió en los albores de la ciencia que "no debía guiarse por lo que veía en la figura, ni tampoco por el mero concepto (Begriffe) de ella, para aprender, por decirlo así, las propiedades de ella; sino que debía producirlas por medio de aquello que él mismo introducía a priori con el pensamiento

I3I Cf. Kant, Immanuel, Crítica de la razón pura, traducción, estudio preliminar y aparato de notas de Mario Caimi, México D.F.: Fondo de Cultura Económica, 2009, BI6I. En adelante CRP, seguido de la numeración correspondiente. 
según conceptos (Begriffen) y exhibía (por construcción) en ella; y que, para conocer con seguridad algo a priori, no debía atribuirle a la cosa nada más que lo que se seguía necesariamente de aquello que él mismo había puesto en ella según su concepto". Lo que convierte, entonces, a la geometría, según Kant, en una ciencia, esto es, en una disciplina que determina a priori sus objetos y que convierte al espacio único en objeto de intuición, es el modo específico y particular de construcción de los conceptos geométricos (un tipo de construcción que produce, por ejemplo, el concepto de triángulo isósceles completamente a priori), lo que, a su vez, depende de la intuición donde se los construye (la intuición pura del espacio). Es tarea de la filosofía comprender en qué se fundamenta la apodicticidad de la matemática para comprender cómo es posible la apodicticidad y sinteticidad de las ciencias en general.

Estas afirmaciones extremadamente sintéticas y preliminares acerca de la geometría dentro de la Crítica nos deben generar ciertas preguntas: ¿cómo es posible representarse al espacio como objeto?; ¿qué significa, exactamente, construir a priori los conceptos geométricos en el espacio puro?, iqué es lo que produce este modo particular de construir los conceptos?, es decir, ¿por qué lo que produce la geometría es matemática?; y, finalmente, icómo se puede argumentar a favor de la universalidad de la matemática a partir de estas consideraciones? Aquí intentaremos responder a estas preguntas. Las respuestas nos darán un bosquejo acerca de lo que se entiende en la obra de Kant por "geometría" y, con ello, también cuáles son sus problemas.

\section{§ 2.}

Para Kant, la geometría, así como el álgebra y la aritmética, es una disciplina matemática ${ }^{133}$. Como tal, entonces, es una disciplina que contiene proposiciones y juicios sintéticos a priori ${ }^{134}$. El procedimiento especial que caracteriza a la geometría produce, por tanto, juicios sintéticos a priori. Sin embargo, ien qué consiste el carácter sintético de los juicios geométricos y cómo estos son a priori?

133 Cf. ibid., BI4-I5.

134 Cf. ibid., BI6, 39, 40, 4I, 63-64. 
Todo juicio geométrico, según Kant, es sintético; esto significa que en ese juicio al sujeto se le enlaza un predicado que originalmente no se encontraba en el sujeto mismo, es decir, los juicios geométricos no son analíticos. Por ejemplo, Kant cree que la proposición "la línea recta es la más corta entre dos puntos" no es una proposición analítica, pues el concepto recta no contiene nada de cantidad, sino solamente de cualidad; es decir, el concepto recta solo indica la forma de una línea (cualidad), pero no su tamaño (cantidad). El concepto más corto es un añadido complementario al de recta, el cual, a su vez, no se extrae analizando el sujeto ${ }^{135}$. Al ser una proposición sintética, y por estar apoyada en una intuición pura ${ }^{136}$, esta proposición es pensada con necesidad y universalidad. Todo principio geométrico, por tanto, es aplicable al mundo con exactitud y la experiencia no puede demostrar lo contrario. Por eso, las verdades de la geometría se pueden conocer independientemente de la experiencia ${ }^{137}$. Hasta aquí el núcleo de la teoría kantiana de la geometría. Pero, ¿cuál es el fundamento de los juicios geométricos de modo que les permita ser, en primer lugar, sintéticos, $y$, en segundo lugar, a priori?

En B74I, Kant explica el procedimiento matemático de construcción de conceptos, el mismo método que el primer geómetra griego descubrió en los albores de la ciencia matemática. Construir un concepto es "exhibir a priori la intuición que le corresponde". Esto significa que al construir un concepto geométrico, el de triángulo isósceles por ejemplo, debemos ir primero de ese mismo concepto a la intuición que le corresponde para luego examinar el concepto de triángulo ahí, in concreto ${ }^{138}$, en la intuición, y estudiar cómo ha sido construido y qué se le puede añadir; es decir, debemos estudiar el concepto de triángulo ahí donde se encuentra. ¿Cuál es este lugar en el cual un concepto geométrico se encuentra in concreto?

\footnotetext{
135 Cf. ibid., BI6.

136 Cf. ibid.

I37 Cf. Kitcher, P., “Kant and Mathematics”, en: Posy, C. J. (ed.), Kant's Philosophy of Mathematics, Springer: Dordrecht, 1992, p. II3.

I38 Cf. Kant, Immanuel, Prolegómenos a toda metafísica futura que haya de poder presentarse como ciencia, traducción de Mario Caimi, Madrid: Istmos, 1999, p. 28I. De ahora en adelante se citará esta obra como Prolegómenos.
} 
En el caso de la geometría, este lugar en el cual los conceptos se encuentran, dice Kant, es una intuición no empírica, es decir, una intuición pura del espacio $^{139}$. En la Exposición metafísica del espacio, Kant explica la naturaleza y las características de esta intuición que permiten que un concepto se encuentre ahí. El espacio es una intuición a priori. En tanto intuición, y no en tanto concepto discursivo, presenta algo inmediato y singular: la representación del espacio. El espacio es intuido como I) la forma de los fenómenos externos ${ }^{140}$ y como 2) un espacio omniabarcador que contiene diversos espacios (limitaciones de la representación intuitiva del espacio único ${ }^{141}$ ). Solo en esta representación ${ }^{142}$, dice Kant, se pueden pensar los diversos y múltiples espacios. Estos múltiples espacios, limitaciones de la representación singular, son los conceptos geométricos, v.gr. el triángulo isósceles es una determinación o limitación específica de la representación espacial. Por eso, como solo en el espacio se pueden pensar los diversos espacios o conceptos, la intuición del espacio sirve de fundamento de todo concepto geométrico $y$, consecuentemente, de toda nueva construcción de un concepto geométrico.

Una vez que hemos visto qué es esta intuición a priori en la cual se exhibe el concepto geométrico, volvamos sobre cómo se construye un concepto geométrico y cómo se intuye tanto a este como al espacio. Todo concepto geométrico, es decir, no discursivo, se construye en el espacio y, en tanto se encuentra ahí, se construye intuyéndolo. Pero en este proceso se construye el significado del concepto en la forma de una exhibición de la figura geométrica, es decir, la construcción es un proceso ostensivo por el cual se determina el significado ${ }^{143}$ de un concepto ${ }^{144}$. Por ejemplo, yo construyo la figura de un determinado triángulo, el isósceles, a priori en el espacio y el significado de ese concepto es conocido por mí como el resultado mismo del proceso, pues el significado de ese triángulo es el resultado de una "síntesis arbitraria". Cuando uno "dibuja" a priori el triángulo isósceles, uno sintetiza la figura triangular con

139 Cf. Kant, Immanuel, CRP, B39.

140 Cf. ibid., B42.

14I Dejaremos para la segunda parte del trabajo la característica de magnitud infinita.

142 "Nur in ihm gedacht werden" (ibid., B39).

143 Cf. ibid., B757.

I44 Cf. Shabel, L., “Kant's Philosophy of Mathematics”, en: Guyer, Paul (ed.), The Cambridge Companion to Kant and Modern Philosophy, Cambridge: Cambridge University Press, 2006, p. 100. 
los conceptos 'dos líneas rectas' y 'línea recta singular'; la imaginación lleva a la unidad estos conceptos geométricos que se encuentran en el espacio, pero que no se encuentran incluidos en el concepto 'triángulo' (en tanto figura de tres lados solamente), y exhibe un nuevo concepto. El proceso, entonces, es una síntesis definitoria que exhibe el contenido como algo nuevo ${ }^{145}$. El resultado es un nuevo concepto, singular e intuido, que se encuentra en la representación a priori del espacio.

Para Kant, el proceso constructivo propio de la geometría, fundado en la intuición pura del espacio, permite enlazar a un determinado sujeto un predicado. Lo que permite el carácter sintético de los juicios geométricos, por tanto, es el proceso de construcción ostensivo. Kant defiende la tesis de que el carácter sintético de los juicios geométricos se alcanza mediante este proceso creativo en A716/B744 utilizando la prueba de Euclides sobre la proposición 32 de los Elementos (la suma de los ángulos interiores de un triángulo plano es igual a dos ángulos rectos: $\left(80^{\circ}\right)^{146}$.

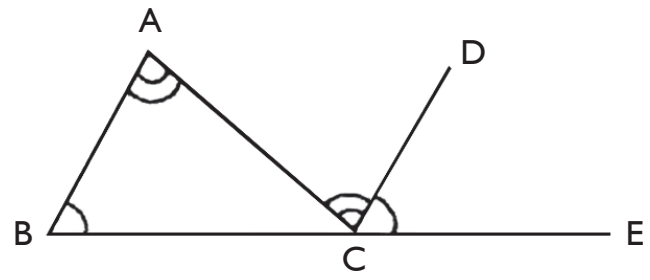

Según Kant, esta representación esquemática de la proposición 32 de Euclides no se alcanza mediante un análisis conceptual; por ejemplo, analizando el concepto de triángulo, el filósofo jamás alcanzará a conocer a priori la proposición euclidiana. En cambio, el geómetra debe extender un lado del triángulo $(B C)$ y extenderlo hasta otro determinado $(C E)$ para alcanzarla. La suma del ángulo $A C E$ será igual a la suma de $C A B$ y $A B C$. Por lo tanto, $A B C, B C A, C A B$ suman juntos dos ángulos rectos. Pero para que esta última proposición sea demostrada y confirmada es necesario trazar $C D$, una línea paralela a BA, lo

145 Cf. Kant, Immanuel, CRP, B757.

146 Harper, W., "Kant on Space, Empirical Realism and the Foundations of Geometry", en: Posy, 
que implica recurrir a las proposiciones euclidianas 3I, 29, I3 y el axioma $2^{147}$. En esa medida, el geómetra, a diferencia del filósofo, debe construir paso a paso el triángulo sintetizándolo con otras partes del espacio, otras líneas, por ejemplo CE y CD, y extrayendo ${ }^{148}$ las conclusiones que se infieran de esta síntesis: por ejemplo, que la suma de los ángulos interiores es igual a dos ángulos rectos. Las líneas $C E$ y $C D$ no se encuentran originalmente en el triángulo primitivo, por tanto, un análisis del triángulo original no muestra la verdad de la proposición 32 o el hecho que el sujeto no se encuentre inicialmente en el predicado. Mediante un proceso deductivo se descubre la función sintética del teorema, v. gr., la validez del teorema mismo ${ }^{149}$.

El carácter sintético de la geometría se funda en la representación intuitiva del espacio sobre el cual se construye un nuevo concepto y el conocimiento del geómetra acerca de las condiciones que el espacio delimita para toda construcción. El espacio es el principio que determina y condiciona la posibilidad y la naturaleza de cualquier tipo de síntesis futura, lo que significa que las síntesis están condicionadas por el tipo de espacio que Kant presupone. Esta concepción del espacio es euclidiana ${ }^{150}$. Cualquier síntesis se da, entonces, en un espacio euclidiano y este tipo de espacio determina a priori lo que se puede sintetizary lo que no. Por ejemplo, si el espacio es euclidiano, y decimos que el espacio solo tiene tres dimensiones ${ }^{151}$, cuando construimos una figura localizando sus líneas de manera específica, sin importar cuál se forme esta será una figura tridimensional; si el espacio que la abarcase fuese distinto, minkowskiano por ejemplo, el resultado sería diferente.

Pero, si bien es posible construir a priori en la imaginación una determinada figura geométrica y alcanzar su verdad también de manera a priori, pues la

147 Cf. The Thirteen Books of Euclid's Elements, I-II, traducción de T.L. Heath, New York: Dover Publications, 1956, pp. 316-318.

148 Cf. Kant, Immanuel, CRP, B4I.

149 Cf. Shabel, L., op. cit., p. 107.

I50 El argumento acerca de la construcción a priori de los conceptos geométricos y la tesis de que el espacio determina la construcción de los conceptos, junto con los ejemplos que Kant presenta, parecen indicar que la representación del espacio es fuertemente euclidiana. Sobre este problema volveremos al final del trabajo. Por lo pronto, me limito a sostener la siguiente tesis: que la representación del espacio para Kant es fuertemente euclidiana. Esta tesis será, en la última sección, matizada. Para una argumentación a favor de esta tesis, cf. Kim, J., "Concepts and Intuitions in Kant's Philosophy of Geometry”, en: Kant-Studien, 97 (2006), pp. I38-I62.

I5I Cf. Kant, Immanuel, CRP, B4I. 
construcción descansa en la intuición pura del espacio, icómo es posible, tal como afirma Kant, que uno tenga también una intuición del espacio -lo que llama "ir del concepto a la intuición que le corresponde"- para determinar la validez de nuestra construcción o síntesis arbitraria? En los Prolegómenos, Kant sostiene que para darle significado y sentido (Bedeutungund y Sinn) a los conceptos del entendimiento se requiere de un cierto uso en concreto de ellos, es decir, de que tengan una cierta aplicación en alguna intuición. La pregunta que Kant se hace allí con respecto a la intuición a priori es "icómo puede la intuición del objeto preceder al objeto mismo?"152, esto es, icómo podemos tener una intuición de algo que no tiene un objeto? La respuesta de Kant es que podemos intuir a priori solo la forma de la intuición sensible ${ }^{153}$. En las Conclusiones a partir de los conceptos precedentes en la Estética ${ }^{154}$, Kant sostiene que el espacio es la forma de todos los fenómenos intuidos por el sentido externo. Tener una intuición del espacio significa tener una intuición de una intuición-forma, de algo que no tiene materia. El resultado, por tanto, de tener como objeto de intuición a una intuición es sui generis: se trata de un objeto intuido como ningún otro, en tanto forma y materia son pura intuición ${ }^{155}$.Cuando construimos a priori en la imaginación una determinada figura geométrica en el espacio, lo que hacemos es delimitar específicamente el espacio, una parte del todo. Esto significa que cuando construimos una figura enfocamos nuestra atención sobre el espacio considerado como una parte del todo; un todo, sin embargo, que en tanto infinito e indeterminado, permite que cada parte sea igual a las otras e igual al todo ${ }^{156}$. Por lo tanto, cuando hacemos geometría estamos codificando ${ }^{157}$ y traduciendo las características del espacio mediante formas, figuras y cantidades determinadas. Podemos, entonces, convertir en objeto de intuición al espacio en sí cuando construimos una figura, cuando lo delimitamos, como por el rabillo del ojo, haciendo visibles y llevando a conciencia, de manera reflexiva y oblicua, el espacio en sí mismo. Cuando hacemos geometría, de esa forma, tenemos un

152 Kant, Immanuel, Prolegómenos, p. 282.

153 Cf. ibid., p. 283.

I54 Cf. Kant, Immanuel, CRP, B42.

I55 Cf. Kitcher, P., op. cit., p. II4.

I56 Cf. Kant, Immanuel, CRP, B40; cf. Kant, Immanuel, Prolegómenos, p. 286.

I57 Cf. Shabel, L., "The Transcendental Aesthetic", en: Guyer, Paul (ed.), The Cambridge Companion to Kant's Critique of Pure Reason, Cambridge: Cambridge University Press, 2010, p. 108. 
doble objeto: el objeto específico, el triángulo isósceles por ejemplo, que está en una intuición, pero al mismo tiempo el espacio mismo, pues el triángulo se construye en el espacio y sobre el espacio en la forma de una restricción concreta $^{158}$. Los conceptos geométricos tienen significado y sentido cuando se hace un uso concreto de ellos, en la intuición, pero este uso concreto produce que la intuición, a su vez, pueda ser objeto de intuición o de conocimiento, que podamos conocer los rasgos del espacio.

Cuando construimos el triángulo de esa forma, sea en la imaginación, sea en un papel, pero siempre de manera a priori, lo que tenemos es un esquema que, si bien es un objeto singular, sin embargo, resulta ser también una representación universal. Pero, ¿qué tipo de esquema es este que produce la imaginación? En el Esquematismo Kant explica que los conceptos puros del entendimiento son completamente heterogéneos con las intuiciones empíricas ${ }^{159}$. Para que los conceptos puros del entendimiento puedan ser aplicados a las intuiciones empíricas es necesario que exista un tercer término que medie entre las representaciones empíricas y las puras conceptuales, por ser homogéneo a cada uno de estos. A este tercer término Kant lo llama "esquema", el cual es un producto de la imaginación. El esquema es la mediación que permite aplicar las categorías del entendimiento a la experiencia. Pero en el caso de la geometría, el entendimiento no parece introducir ningún concepto que sea relevante para el tipo de cognición de la geometría, ya que los conceptos de la geometría no nacen de la actividad espontánea del entendimiento, sino de la intuición pura del espacio, los cuales son producidos por la imaginación. Esta diferencia sustancial causa que los esquemas o conceptos puros geométricos de la imaginación se encuentren en una relación de homogeneidad con los conceptos empíricos. Por ejemplo, el concepto empírico de plato tiene homogeneidad con el concepto geométrico de círculo. Lo que significa que la redondez del plato puede ser medida y calculada matemáticamente ${ }^{160}$. El esquema geométrico, lo que produce la imaginación, no resulta ser, a diferencia de los esquemas de los conceptos puros del entendimiento, una representación mediadora; la imaginación no produce una imagen sensible geométrica, la cual sería producto de la facultad empírica de la imaginación. El propósito

158 Cf. Kitcher, P., op. cit., p. II5.

I59 Cf. Kant, Immanuel, CRP, BI76-I78.

160 Sobre esto volveremos inmediatamente. 
de la actividad de la imaginación con respecto a los esquemas de la geometría es llevar a unidad la determinación de la sensibilidad a priori' ${ }^{161}$, es decir, las determinaciones que realizamos al espacio. El esquema es, pues, una "regla de la síntesis de la imaginación, con respecto a figuras puras en el espacio"162. Su aplicabilidad universal reside, como indica Shabel ${ }^{163}$, no a causa del hecho de que el esquema construido sea individual y universal, sino en la conciencia de que la construcción se ciñe a las reglas de la síntesis en el espacio puro. Cuando construimos una figura geométrica a priori, construimos una figura, sostiene Kant, cuya aplicabilidad es universal, pues no depende de ninguna consideración empírica, sino en el hecho de que la intuición en la cual se funda es también la forma de todos los fenómenos externos y esa forma establece una regla de aplicación en la experiencia ${ }^{164}$. Por eso, cuando construimos una figura geométrica prestando atención a las reglas de la síntesis de la imaginación, poco importan las determinaciones específicas y concretas de la figura construida, por ejemplo, el tamaño del trazo en la arena del cuadrado o la longitud de la línea imaginada en el caso de la proposición 32 de Euclides.

Al construir un concepto geométrico tenemos acceso, porque lo convertimos en objeto de nuestra intuición, a las condiciones cognitivas que regulan y gobiernan el proceso de construcción mismo: la intuición pura del espacio. Saber qué es el espacio como intuición pura nos permitirá comprender cómo entiende Kant que las proposiciones o juicios geométricos sean, además de sintéticos, también a priori.

\section{§ 3.}

¿Qué significa que los juicios de la geometría sean a priori? "A priori” significa estricta universalidad y necesidad ${ }^{165}$. Es decir, que los juicios de la geometría, si son sintéticos y construidos en la intuición pura del espacio, son todos

\footnotetext{
161 Cf. ibid., BI79.

162 Ibid., BI80.

163 Cf. Shabel, L., "Kant's Philosophy of Mathematics”, en: Guyer, Paul (ed.), The Cambridge Companion to Kant and Modern Philosophy, p. II2.

164 Cf. Young, J. M., “Construction, Schematism and Imagination”, en: Posy, C. J. (ed.), op. cit., p. 166. 
ellos verdaderos de manera universal y necesaria. Esto quiere decir que la experiencia I) nunca los contradecirá, a saber, que se aplicarán a ella con universalidad, y 2) que ella no es la fuente de su apodicticidad. Según Kant, en la primera edición de la Crítica ${ }^{166}$, lo que permite la apodicticidad de todos los axiomas de la geometría es que el espacio es una intuición pura y no un concepto discursivo. Si el espacio fuese un concepto adquirido a posteriori, supongamos, los axiomas de la geometría solo serían meras percepciones, es decir, proposiciones contingentes, adquiridas inductivamente $y$, naturalmente, no axiomas. La argumentación de Kant acerca del carácter sintético y apodíctico de la geometría descansa en la Exposición metafísica del espacio: éste último es una intuición pura y como tal permite explicar la apodicticidad de la geometría ${ }^{167}$. La Exposición trascendental solo refuerza lo que Kant ya ha explicado en la sección anterior. La representación que se debe presuponer del espacio para que la geometría sea sintética y a priori no puede ser otra que la de una intuición pura ${ }^{168}$. Esta afirmación es presentada de nuevo en el parágrafo 10 de los Prolegómenos: “(...) esta presuposición es absolutamente necesaria, si las proposiciones sintéticas a priori han de ser admitidas como posibles (...)"169. Sin embargo, Kant solo ha explicado la manera cómo se construyen las proposiciones o conceptos geométricos y qué se sigue de ello 170 (su apodicticidad).Pero, icómo explica Kant que sea posible que los juicios sintéticos a priori de la geometría sean aplicables con estricta universalidad al mundo de la experiencia ${ }^{171}$ ?

El argumento acerca de la geometría en la Exposición trascendental sirve para mostrar, y no demostrar ${ }^{172}$, que ella solo es posible si el espacio es una intuición a priori. Pero hacia el final de la Exposición trascendental, Kant comienza a argumentar a favor de la idealidad trascendental/realidad empírica del espacio $^{173}$. Kant sostiene que la intuición pura del espacio es la forma del sentido externo en general. Es decir, los fenómenos exteriores se encuentran ya en

\footnotetext{
166 Cf. ibid., A24.

167 Cf. ibid., B39.

168 Cf. ibid., B40-4I.

169 Kant, Immanuel, Prolegómenos, p. 283.

170 Cf. Kant, Immanuel, CRP, B40.

I7I Cf. ibid., BI96.

172 Esta es la tesis sostenida por Lisa Shabel (cf. Shabel, L., "Kant's 'Argument from Geometry”," en: Journal of the History of Philosophy, XLII, No. 2 (2004), pp.195-215.

173 Cf. Kant, Immanuel, CRP, B4I, B44.
} 
el espacio y, por lo tanto, determinados a priori por ellos. De esto se sigue, inmediatamente, que los aspectos del espacio como intuición pura deben también encontrarse en las representaciones empíricas, en los fenómenos ${ }^{174}$. Sin entrar en detalles acerca de la discusión que entabla Kant con Leibniz y Newton acerca del concepto de espacio, el argumento que Kant construye desde el final de Exposición trascendental hasta las Conclusiones a partir de los conceptos precedentes acerca de la relación que existe entre el espacio como intuición pura y los fenómenos exteriores es como sigue: I) el espacio no representa ninguna propiedad de las cosas en sí, ni es la representación de las relaciones que tienen las cosas mismas entre ellas, ni es independiente de la mente humana; 2) el espacio es la forma de todos los fenómenos externos; 3) es decir, es la condición subjetiva de la sensibilidad; 4) lo que significa que se puede entender cómo la forma de todos los fenómenos se nos da o nos es dada de manera a priori; 5) por lo cual concluimos que a priori podemos determinar, si nos ocupamos de nuestra representación pura del espacio, los principios de las relaciones de los fenómenos ${ }^{175}$.

Si el espacio es, además de ser una intuición pura, la forma subjetiva de todos los fenómenos externos (puntos I, 2 y 3), esto es, si las cosas solo se nos aparecen a nosotros (punto 4) como estando ya en el espacio, entonces el espacio tiene realidad objetiva con respecto a los fenómenos; el espacio se nos muestra en el mundo a través de los fenómenos. Cuando Kant sostiene que el espacio tiene realidad objetiva, lo que quiere afirmar es que los objetos se nos presentan, por ejemplo, a nosotros y para nosotros desde una perspectiva tridimensional y bajo las reglas que el espacio como intuición pura establece. El mundo en sí no está -si lo que determina la representación del espacio es una teoría del espacio euclidiana- sujeto a las reglas y condiciones que el espacio como intuición pura establece; solo se encuentra sujeto a estas reglas el mundo de los fenómenos, es decir, el mundo tal como se le aparece a un sujeto es espacial (lo que equivale a decir tridimensional ${ }^{176}$ ). En tanto algo es un fenómeno y no una cosa en sí, se encuentra necesariamente sometido a

I74 Cf. Falkenstein, L., Kant's Intuitionism: A Commentary on the Transcendental Aesthetic, Toronto: University of Toronto Press, 1995, p. 200.

175 Cf. Kant, Immanuel, CRP, B42.

176 Cf. ibid., B43. Esto podría entenderse también como euclidiano. Sobre este problema, revísese la última sección. 
las leyes del espacio ${ }^{177}$. Los objetos geométricos y los fenómenos externos, de esa forma, comparten un territorio común: el espacio como intuición pura. Solo así podemos comprender la afirmación en el Esquematismo de que los conceptos geométricos y los conceptos empíricos son homogéneos: ambos objetos están sometidos a las mismas leyes que el espacio determina; ambos objetos, por tanto, exhiben, aunque de manera distinta -uno, el esquema, de manera pura, y el otro, el fenómeno, de manera circunstancial y perceptiblemente- las características del espacio. Pero no solo ambos tipos de objetos, entes matemáticos y fenómenos, están sometidos a las mismas leyes. Ambos tipos de objetos son susceptibles de ser cuantificados y medidos, pues el espacio es representado como una cantidad (quanta) infinita dada ${ }^{178}$, es decir, su misma naturaleza posibilita que los fenómenos también sea geométricamente definibles; lo que equivale a que la matemática pueda ser aplicable de manera universal y necesaria ${ }^{179}$. La construcción matemática de un concepto geométrico en la intuición pura del espacio, sea en la imaginación, sea en un papel, hace, entonces, del espacio su objeto. Cuando se construye un concepto singular a priori, y no se lo extrae a posteriori, se forma un patrón o un esquema que exhibe las mismas condiciones o características generales que los fenómenos exhiben por estar en el espacio ${ }^{180}$; características que son, por igual, las del espacio mismo.

\section{§ 4.}

Hasta aquí, la aplicación universal a la experiencia de las proposiciones de la geometría ha sido argumentada por el mismo Kant a partir solamente de las explicaciones exhibidas en la Estética trascendental. Este camino argumentativo, el recurrir solo a la Estética, parece excluir implícitamente al entendimiento de tomar parte en el proceso, no solo de la construcción de conceptos geométrico, pues es la imaginación quien lleva a cabo esta labor, sino además de la justificación de la aplicabilidad de los juicios sintéticos a priori de la geometría a los fenómenos, dado que la justificación reside en el argumento de la idealidad

177 Cf. Harper, W., op. cit., pp. 268-269.

178 Cf. Kant, Immanuel, CRP, B39.

179 "Lo que la geometría dice de esta [v. gr. del espacio puro], vale también sin objeción para aquella [v. gr. la experiencia]" (ibid., B206).

180 Cf. Kant, Immanuel, Prolegómenos, p. 288. 
trascendental del espacio. De hecho, en los Prolegómenos, Kant sigue este último camino, es decir, el de justificar la aplicabilidad de la geometría en la trascendentalidad del espacio: "puesto que el espacio, tal como lo piensa el geómetra, es precisamente la forma de la intuición sensible, que encontramos a priori en nosotros y que contiene el fundamento de la posibilidad de todos los fenómenos externos (según su forma), estos [v. gr., los fenómenos] deben concordar necesariamente y con toda precisión con las proposiciones del geómetra"|81. En la Analítica trascendental Kant repite este argumento, pero explicita porqué el entendimiento no toma parte en la construcción de los axiomas de la geometría: los principios de la matemática no forman parte del sistema de los principios del entendimiento puro, pues se extraen de la intuición pura del espacio y no del entendimiento; tampoco puede demostrarse la evidencia de los juicios geométricos mediante la aplicación del entendimiento, ya que la evidencia de la matemática descansa únicamente en la intuición pura ${ }^{182}$. Cuando en el Principio supremo de todos los juicios sintéticos ${ }^{183}$ Kant formula el Axioma de la intuición ("todas las intuiciones son cantidades extensivas"), quiere demostrar que las ciencias de la naturaleza -pensemos en la física por ejemplo-, en tanto aplicación de las categorías, v. gr., de la cantidad, a la experiencia, presuponen y requieren la posibilidad a priori de la matematización o cuantificación de los fenómenos, esto es, presuponen que los fenómenos exhiban las características del espacio.

\section{§ 5.}

La importancia de estudiar la teoría kantiana de la geometría no radica exclusivamente en que ella, en tanto disciplina matemática, determina a priori su objeto, y por tanto sirve de ejemplo a la filosofía, sino en que a causa del objeto mismo, el espacio como intuición pura, nos obliga a estudiar qué entiende Kant por sensibilidad y espacio. Para Kant, el estudio de la sensibilidad lo obliga a repensar qué se entiende por espacio y, con ello, qué se entiende por geometría. Cuando analizamos la teoría kantiana de la geometría,

181 lbid.

182 Cf. Kant, Immanuel, CRP, BI88-I89.

183 Cf. ibid., BI93. 
entonces, lo que realmente hacemos es pasar revista a la teoría kantiana de la sensibilidad y viceversa.

Sin duda, la teoría kantiana de la geometría no se encuentra libre de problemas. Estos van desde la aparición en el siguiente siglo de teorías geométricas noeuclidianas; la factibilidad de intuiciones infinitas del espacio ${ }^{184}$; la posibilidad misma de la intuición del espacio; la no diferenciación entre geometría pura, es decir, exclusivamente deductiva, y geometría física o mecánica aplicada ${ }^{185}$; y el papel del entendimiento con respecto a la creación de conceptos geométricos. El análisis de estos problemas sería objeto de otra investigación. Todos estos problemas parecen representar ataques no solo serios y comprometedores, sino mortales a la teoría de la geometría en Kant y, con ello, a la teoría de la sensibilidad. Pero, como ya hemos visto, valdría la pena preguntarse y distinguir cuáles de estas refutaciones provienen desde una visión positivista de la ciencia, la cual parece suponer que una vez superada científicamente esta teoría geométrica, filosóficamente ya no tiene nada que decirnos, y cuáles no.

Enúltima instancia, la valoración que tengamos de la teoría kantiana dependerá de la perspectiva con la cual la estudiemos. A pesar de las innovaciones que la teoría kantiana de la geometría representa con respecto a sus antecesores, Leibniz y Newton, en sí misma es insostenible si la pensamos como fuertemente comprometida con la teoría euclidiana del espacio ${ }^{186}$. De hecho, la tesis kantiana a favor del carácter sintético de la geometría como resultado de la construcción de conceptos geométricos por la imaginación parece descansar en una concepción del espacio euclidiana, pues no solo los ejemplos de ellos son euclidianos, sino que el concepto está sujeto a ciertas reglas específicas de síntesis. Reglas que solo una teoría del espacio, sea cual fuese, pero una teoría específica, determinaría. Si, además de estos argumentos, consideramos la teoría euclidiana como superada y abandonada por las teorías no-euclidianas,

I84 Desde la publicación misma de la Crítica de la razón pura se viene discutiendo si el espacio puede ser representado o no como una cantidad infinita dada (cf. ibid., B39). Sobre este punto específico, cf. Patton, L., "The Paradox of Infinite Given Magnitude: Why Kantian Epistemology Needs Metaphysical Space”, en: Kant-Studien, 102 (20II), pp. 273-289.

I85 Sobre este punto, ver el excelente resumen que hace Stephen Barker sobre los ataques a la teoría kantiana desde la geometría no-euclidiana (cf. Barker, Stephen, "Kant's view of Geometry: A Partial Defense", en: Posy, C. J. (ed.), op. cit., pp. 22I-243).

I86 Cf. Höffe, O., Kant's Critique of Pure Reason: The Foundation of Modern Philosophy, Springer: Dordrecht, 2009, p. 105. 
entonces, la única posible solución parecería ser considerar la teoría kantiana de la geometría como cosa del pasado. Ahora bien, si la pensamos, en cambio, histórica y filosóficamente, y reducimos la teoría kantiana a la Estética trascendental, la teoría kantiana de la geometría sigue siendo relevante, pues fenoménicamente el mundo se nos presenta como euclidiano. Si la geometría al hacerse empírica debe transformar los presupuestos intuitivos de la geometría euclidiana (que el espacio es tridimensional), esto solo mostraría que el espacio en tanto trascendental no es solamente euclidiano. Esta posición debilita el carácter euclidiano de la geometría en Kant, pero deja intacto su fundamento intuitivo puro y trascendental ${ }^{187}$. Sin embargo, este tipo de argumentación, la cual intenta salvar la idealidad trascendental del espacio a costas de reducir el carácter euclidiano implícito de la teoría kantiana, debilita, si no contradice abiertamente, la tesis kantiana misma de que es la intuición pura la que determina el carácter del espacio externo y no al revés, pues delimitaría el campo de acción del espacio a la mera experiencia intuitiva y cotidiana y no al espacio en sí mismo; es decir, la universalidad de la geometría sería reducida a la experiencia espacial mundana que experimentan los seres humanos diariamente sin geometrizarla. Además, estudios realizados a mediados del siglo pasado parecen demostrar que percibimos el mundo de manera hiperbólica y no euclidianamente ${ }^{188}$. Sería, finalmente, la experiencia misma la que determinaría nuestras nuevas y diferentes representaciones del espacio, esas no-euclidianas. Quizás, la mejor defensa consistiría en sostener, con Höffe, que el espacio en sí mismo no debe ser ni euclidiano ni no-euclidiano. La prueba de esto radicaría en que Kant jamás incluye ningún argumento o axioma euclidiano dentro de la exposición trascendental del espacio. Que la representación del espacio sea una magnitud no la convierte necesariamente en euclidiana, sino solo en matemática. Esta salida, no obstante, no toma en cuenta los argumentos kantianos sobre el tipo de construcción de los conceptos geométricos.

En última instancia, como se puede ver, sea cual sea la perspectiva que adoptemos ninguna parece encontrarse libre de problemas. Parece que siempre es posible contraargumentar cualquier posible defensa a la geometría kantiana. La única conclusión verdadera y libre de mayores problemas sería que la íntima conexión entre geometría y la teoría kantiana del fenómeno es lo que la hace a la primera aún relevante en tanto objeto de estudio en la obra de Kant.

187 Cf. ibid., p. 110.

72 I88 Cf. Trudeau, R., op. cit., pp. 25I-252. 